\title{
Analytic Approach to Small $x$ Structure Functions
}

\author{
J.R. Forshaw, R.G. Roberts and R.S.Thorne \\ Rutherford Appleton Laboratory, \\ Chilton, Didcot OX11 0QX, England.
}

\begin{abstract}
We present a method for the analytic solution of small $x$ structure functions. The essential small $x$ logarithms are summed to all orders in the anomalous dimensions and coefficient functions. Although we work at leading logarithmic accuracy, the method is general enough to allow the systematic inclusion of sub-leading logarithms. Results and predictions are presented for the gluon density, and the structure functions $F_{2}\left(x, Q^{2}\right)$ and $F_{L}\left(x, Q^{2}\right)$. We find that corrections to the simple double logarithmic calculation are important in the HERA range and obtain good fits to all available data.
\end{abstract}


As a result of the recent work of Catani and Hautmann [1], it is now possible to include the dominant small $x$ dynamics encompassed by the formalism of Balitsky, Fadin, Kuraev and Lipatov (BFKL) [2] within the framework of the renormalisation group and collinear factorisation, and some (mostly numerical) studies have already been performed [3, 4]. In this paper, we wish to present an analytic solution to the relevant evolution equations and their convolution with the appropriate coefficient functions. Throughout we work in the high energy limit, i.e. we sum all terms in the perturbative expansion of the cross section which are

$$
\sim\left(\alpha_{s} \ln \frac{s}{Q^{2}}\right)^{n}
$$

where $s$ is the relevant centre-of-mass energy and $Q^{2}$ characterises the typical short distances involved. We shall focus on deep inelastic scattering at the DESY ep collider, HERA. In which case, $\sqrt{s}$ is the $\gamma p$ centre-of-mass energy and $-Q^{2}$ is the photon virtuality, i.e. the Bjorken- $x$ variable, $x \approx Q^{2} / s$. Our approach is quite general and it will be clear how to extend it beyond the leading logarithmic accuracy.

\section{Altarelli-Parisi Evolution at small $x$ and the gluon density}

Recall the Dokshitzer, Gribov, Lipatov, Altarelli, Parisi (DGLAP) equations for the parton distribution functions [5]:

$$
\frac{\partial f_{N}^{i}\left(Q^{2}\right)}{\partial \ln Q^{2}}=\sum_{j} \gamma_{N}^{i j} f_{N}^{j}\left(Q^{2}\right) .
$$

$f_{N}^{i}\left(Q^{2}\right)$ is the Nth moment of the momentum distribution for partons of type $i$ and $\gamma_{N}^{i j}$ is the anomalous dimension matrix, i.e.

$$
\begin{aligned}
f_{N}^{i}\left(Q^{2}\right) & =\int_{0}^{1} d x x^{N-1} f^{i}\left(x, Q^{2}\right), \\
\gamma_{N}^{i j} & =\int_{0}^{1} d x x^{N} P_{i j}(x) .
\end{aligned}
$$

Our notation is such that the important gluon anomalous dimension,

$$
\gamma_{N}^{g g}=\frac{\bar{\alpha}_{s}}{N}+2 \zeta(3)\left(\frac{\bar{\alpha}_{s}}{N}\right)^{4}+\ldots
$$

and $\bar{\alpha}_{s}=3 \alpha_{s} / \pi$.

These equations are solved given the boundary conditions, $f_{N}^{i}\left(Q_{0}^{2}\right)$, i.e. they allow the $Q^{2}$-dependence of the parton distribution functions to be determined but not their absolute normalisation. 
In the high energy (i.e. small $x$ ) limit, we keep only those terms in the anomalous dimension matrix which are $\sim\left(\alpha_{s} / N\right)^{n}$, i.e. the leading logarithmic terms in the splitting functions. In this case, evolution is driven by $\gamma_{N}^{g g}$, which satisfies [2, 6]

$$
1=\frac{\bar{\alpha}_{s}}{N} \chi\left(\gamma_{N}^{g g}\right)
$$

where

$$
\chi(\gamma)=2 \psi(1)-\psi(\gamma)-\psi(1-\gamma)
$$

and $\psi(\gamma)$ is the Euler-gamma function. The first two non-zero terms in the series expansion are written in eq.(3). The DGLAP equations then have the simple solution:

$$
\begin{aligned}
f_{N}^{s}\left(Q^{2}\right) & =f_{N}^{s}\left(Q_{0}^{2}\right) \\
f_{N}^{g}\left(Q^{2}\right) & =\left[f_{N}^{g}\left(Q_{0}^{2}\right)+\frac{4}{9} f_{N}^{s}\left(Q_{0}^{2}\right)\right] \exp \left(\int_{Q_{0}^{2}}^{Q^{2}} \frac{d k^{2}}{k^{2}} \gamma_{N}^{g g}\right)-\frac{4}{9} f_{N}^{s}\left(Q_{0}^{2}\right)
\end{aligned}
$$

The singlet quark density is $f_{N}^{s}\left(Q^{2}\right)=\sum_{i} f_{N}^{i}\left(Q^{2}\right)$ where the sum runs over all quarks and anti-quarks. Since we work in the small $x$ region, we expect the gluon density to be dominant and subsequently drop all reference to the singlet density (except implicitly in the input to $\left.F_{2}\left(x, Q^{2}\right)\right)$. We have explicitly checked that this makes very little quantitative difference to our results.

In order to construct a sensible gluon structure function, we do not merely invert the $N$ space solution above. It is more natural to define the gluon structure function to be that object which would be observed if we had a coloured current available as our probe. In which case there are important contributions which arise, not only from the QCD evolution but also from the coefficient function. One can think of such corrections as arising from graphs which should not be exponentiated via the renormalisation group and so contain no explicit strong ordering of the rung momenta. These graphs are essential for a sensible definition of the gluon structure function (e.g. as the object which is closely related to the structure function $F_{L}\left(x, Q^{2}\right)$ ) and for consistency with the structure function which is constructed by integrating the "unintegrated gluon density', $\mathcal{F}\left(x, k^{2}\right)$, obtained by solving the BFKL equation [7]. To see this, we start from the BFKL definition of the gluon structure function, i.e.

$$
G\left(x, Q^{2}\right)=\int_{0}^{\infty} \frac{d k^{2}}{k^{2}} \int_{x}^{1} \frac{d z}{z} \delta(1-x / z) \Theta\left(Q^{2}-k^{2}\right) \mathcal{F}\left(z, k^{2}\right) .
$$

The hard scatter cross section which is to be convoluted with $\mathcal{F}\left(x, k^{2}\right)$ is thus

$$
\hat{\sigma}_{N}\left(k^{2} / Q^{2}\right)=\int_{0}^{1} d x x^{N-1} \delta(1-x) \Theta\left(Q^{2}-k^{2}\right) .
$$


Now, in the formalism of Catani and Hautmann [1],

$$
G_{N}\left(Q^{2}\right)=\gamma_{N}^{g g} \int_{0}^{\infty} \frac{d k^{2}}{k^{2}}\left(\frac{k^{2}}{Q^{2}}\right)^{\gamma_{N}^{g g}} \hat{\sigma}_{N}\left(k^{2} / Q^{2}\right) R_{N} f_{N}^{g}\left(Q^{2}\right)
$$

i.e.

$$
G_{N}\left(Q^{2}\right)=R_{N} f_{N}^{g}\left(Q^{2}\right)
$$

Here $R_{N}$ plays the role of a coefficient function for the 'natural' definition of the gluon structure function [7, 8]. This important factor, which is a process independent but scheme dependent quantity is given, in the $\overline{\mathrm{MS}}$ scheme (which we shall employ throughout this paper) by

$$
\begin{aligned}
R_{N}= & \left\{\frac{\Gamma\left(1-\gamma_{N}^{g g}\right) \chi\left(\gamma_{N}^{g g}\right)}{\Gamma\left(1+\gamma_{N}^{g g}\right)\left[-\gamma_{N}^{g g} \chi^{\prime}\left(\gamma_{N}^{g g}\right)\right]}\right\}^{1 / 2} \times \\
& \exp \left\{\gamma_{N}^{g g} \psi(1)+\int_{0}^{\gamma_{N}^{g g}} d \gamma \frac{\psi^{\prime}(\gamma)-\psi^{\prime}(1-\gamma)}{\chi(\gamma)}\right\} .
\end{aligned}
$$

\section{Solution in $x$-space}

Let us now show how to invert the solution for $G_{N}\left(Q^{2}\right)$ back into $x$-space. We must perform the integral

$$
G\left(x, Q^{2}\right)=\frac{1}{2 \pi i} \int d N f_{N}^{g}\left(Q_{0}^{2}\right) R_{N} \exp \left(N \ln 1 / x+Z_{N}\left(Q^{2}\right)\right)
$$

where the integral is over a contour to the right of all singularities, and

$$
\begin{aligned}
Z_{N}\left(Q^{2}\right) & =\int_{Q_{0}^{2}}^{Q^{2}} \frac{d k^{2}}{k^{2}} \gamma_{N}^{g g} \\
& =\frac{\gamma^{2} \zeta}{N}+\sum_{m=2}^{\infty} \frac{a_{m}}{m-1} \frac{\gamma^{2}}{N}\left[\left(\frac{\bar{\alpha}_{s}\left(Q_{0}^{2}\right)}{N}\right)^{m-1}-\left(\frac{\bar{\alpha}_{s}\left(Q^{2}\right)}{N}\right)^{m-1}\right] \\
& =\sum_{m=1}^{\infty} \frac{b_{m}(\zeta)}{N^{m}}
\end{aligned}
$$

$\zeta=\ln \left(\alpha_{s}\left(Q_{0}^{2}\right) / \alpha_{s}\left(Q^{2}\right)\right), \gamma^{2}=12 / \beta_{0}$ and, for consistency with the standard approach, we run the coupling at the scale $k^{2}$ (in the anomalous dimension integral). However, we note that at the leading logarithmic accuracy this is an essentially free choice. The coefficients $a_{n}$ define the series expansion of the gluon anomalous dimension:

$$
\gamma_{N}^{g g}=\sum_{n=1}^{\infty} a_{n}\left(\frac{\bar{\alpha}_{s}}{N}\right)^{n}
$$


We write the factor $R_{N}$ also as a series expansion:

$$
R_{N}=\sum_{n=0}^{\infty} c_{n}\left(\frac{1}{N}\right)^{n},
$$

and we note that $R_{N}=1+\frac{8}{3} \zeta(3)\left(\bar{\alpha}_{s} / N\right)^{3}+\ldots$

We choose the boundary condition,

$$
f_{N}^{g}\left(x, Q_{0}^{2}\right)=\mathcal{N} \Theta\left(x_{0}-x\right),
$$

which becomes $f_{N}^{g}\left(Q_{0}^{2}\right)=\mathcal{N} / N$ in moment space if we choose $x / x_{0}$ as the conjugate variable to $N$. This starting distribution is a good approximation to the expected $\sim(1-x)^{5}$ behaviour if $x_{0} \sim 0.1$, and leads to reliable results for $x \lesssim 0.01$. The choice of a flat starting distribution (at small $x$ ) is motivated by the known behaviour of total cross sections at high energies, i.e. the 'soft' pomeron is known to have intercept close to 1 [9]. It is the small $x$ behaviour one would expect in the absence of any perturbative QCD corrections.

We can now perform the $N$-plane integral by taking a particular choice of contour to be the line from $r-i \infty$ to $r+i \infty$ plus a circle with centre at the origin and radius $r>4 \bar{\alpha}_{s}\left(Q_{0}^{2}\right) \ln 2$. This choice of contour ensures the analyticity of the integrand along the contour, i.e. that all cuts lie within the circle (e.g. for the precise location of the cuts see ref.[四). The value of the integral is now equal to that over the circle, and putting $N=r e^{i \theta}$ we obtain in a straightforward manner:

$$
\begin{aligned}
G\left(x, Q^{2}\right) & =\mathcal{N} \sum_{i=0}^{\infty} c_{i}\left(\frac{\xi}{\gamma^{2} \zeta}\right)^{i / 2} I_{i}(2 \gamma \sqrt{\xi \zeta}) \\
& +\mathcal{N} \sum_{i=0}^{\infty} \sum_{j=2}^{\infty} c_{i} b_{j}\left(\frac{\xi}{\gamma^{2} \zeta}\right)^{(i+j) / 2} I_{i+j}(2 \gamma \sqrt{\xi \zeta}) \\
& +\mathcal{N} \sum_{i=0}^{\infty} \sum_{j=2}^{\infty} \sum_{k=2}^{\infty} \frac{c_{i} b_{j} b_{k}}{2}\left(\frac{\xi}{\gamma^{2} \zeta}\right)^{(i+j+k) / 2} I_{i+j+k}(2 \gamma \sqrt{\xi \zeta})+\ldots \ldots .
\end{aligned}
$$

where $\xi=\ln \left(x_{0} / x\right)$. We note that exactly the same method could be used if we were to choose a powerlike input, or even the $(1-x)^{5}$ behaviour. One simply finds the moment space expression for the input and expands in powers of $N$. We also note that for small $x$ the result obtained using the saddle-point method to evaluate eq.(12) does not give a good approximation and provides misleading results. This failure occurs essentially because, along the contour of steepest decent, the integrand does not fall quickly enough for values of $N$ far from the saddle-point.

Let us now discuss our solution. Firstly, we see explicitly the double log result,

$$
G\left(x, Q^{2}\right) \sim I_{0}(2 \gamma \sqrt{\xi \zeta})
$$


which arises when only the leading order (in $\alpha_{s}$ ) terms are kept. Going beyond this first term, the inverse factorials associated with the Bessel functions ensure that the summations in our expression converge for all $x$, despite the fact that the expansions of $\gamma_{N}^{g g}$ and $R_{N}$ diverge for $N<4 \bar{\alpha}_{s} \ln 2$. This effect of convergence in $x$-space was pointed out using a similar, but slightly less direct, argument in [3]. Using the first sum in eq.(16) we can recover the power behaviour of the structure function at small enough $x$. It arises after taking the small argument expansion of the Bessel function (which is appropriate for large order Bessel functions) and using the fact that $c_{n+1} / c_{n}=4 \bar{\alpha}_{s} \ln 2$ for asymptotically large $n$, i.e. the general term in the sum over $i$ is

$$
\sim\left(4 \bar{\alpha}_{s} \ln 2\right)^{i}\left(\frac{\xi}{\gamma^{2} \zeta}\right)^{i / 2} \frac{\left(\gamma^{2} \zeta \xi\right)^{i / 2}}{i !}=\frac{\left(4 \xi \bar{\alpha}_{s} \ln 2\right)^{i}}{i !}
$$

A similar power behaviour is generated by the other terms in eq.(16), i.e. due to the $Q^{2}$ evolution, but they are not important for all practical values of $x$. The same qualitative conclusion has been reached in ref. [3], where a sum of Bessel functions was presented as an approximate solution for $f^{g}\left(x, Q^{2}\right)$. In other words, the dominant corrections to the double log result are due to the presence of the $R_{N}$ factor, i.e. the corrections to the evolution are small (due to the relatively small size of the coefficients in the expansion of the gluon anomalous dimension), only becoming dominant at very large $Q^{2}$ and/or very small $x$.

The fraction of $G\left(x, Q^{2}\right)$ which arises solely from the double log graphs (i.e. the $I_{0}$ Bessel function) is presented in the contour plot shown in fig.(1). It can be seen that the high energy (BFKL) corrections are significant over the HERA range despite the fact that the coefficients $c_{1}$ and $c_{2}$ vanish. We note that the contribution from the BFKL corrections to the evolution (i.e. those terms involving the $b_{i}$ coefficients) are almost entirely negligible, in fact they contribute less than $4 \%$ over the $x$ - $Q^{2}$ range probed at HERA. In fig.(2), we show the $x$ dependence of $G\left(x, Q^{2}\right)$ at different $Q^{2}$ values and compare to the double log contribution. In all of our plots, we choose $x_{0}=0.1$ and take $\mathcal{N}=1.1$ and $Q_{0}^{2}=2.0 . \mathcal{N}$ and $Q_{0}^{2}$ are the only parameters for the gluon, and are fixed by fitting $F_{2}\left(x, Q^{2}\right)$ to the HERA data (see the following section for a discussion of this procedure). Note that our approach does not permit a flat gluon structure function, even though our input density was flat. This is in keeping with the standard BFKL result developed by direct solution of the BFKL equation. The scale $Q_{0}$ is to be understood as the scale below which we cannot use the perturbative approach. As such, we are unable to make any definite statements regarding the eventual saturation and flattening off of the small $x$ structure functions (as $Q^{2}$ falls below $Q_{0}^{2}$ ) since this procedure is governed by physics beyond 
that which is considered here. (However, we do see a hint of the breakdown of our approach, as we will discuss in the next section.) Indeed, we shall find that the quality of our fits to $F_{2}\left(x, Q^{2}\right)$ is largely insensitive to the choice of $Q_{0}^{2}$ once it is above $\approx 1 \mathrm{GeV}^{2}$. Our approach should be contrasted to that which attempts to evolve from some, typically quite low, value of $Q_{0}^{2}$ with a flat (or valencelike) starting distribution to higher $Q^{2}$ 10, 11.

\section{Deep inelastic structure functions}

In the previous section we concentrated on the gluon structure function, $G\left(x, Q^{2}\right)$. It involves no new techniques to extend the formalism to the case of the deep inelastic structure functions, $F_{2}\left(x, Q^{2}\right)$ and $F_{L}\left(x, Q^{2}\right)$. Eq.(10), which defines the gluon structure function, is merely a specific form of the more general expression for the dimensionless cross section, $F\left(x, Q^{2}\right)$ :

$$
F_{N}\left(Q^{2}\right)=C_{N}\left(Q^{2} / \mu_{F}^{2}\right) f_{N}^{g}\left(\mu_{F}^{2}\right)
$$

where $\mu_{F}^{2}$ is the factorisation scale (chosen to equal $Q^{2}$ ) and $C_{N}\left(Q^{2} / \mu_{F}^{2}\right)$ is the coefficient function (equal to $R_{N}$ in the case of the gluon structure function).

Catani and Hautmann have shown that the coefficient function can be factorised into a product of the process independent (but factorisation scheme dependent) factor, $R_{N}$, and a process dependent factor, $h_{N}\left(\gamma_{N}^{g g}\right)$, where [1]

$$
h_{N}(\gamma) \equiv \gamma \int_{0}^{\infty} \frac{d k^{2}}{k^{2}}\left(\frac{k^{2}}{\mu_{F}^{2}}\right)^{\gamma} \hat{\sigma}_{N}\left(k^{2} / Q^{2}\right) .
$$

The hard subprocess cross section, $\hat{\sigma}_{N}\left(k^{2} / Q^{2}\right)$, is the lowest order (in $\left.\alpha_{s}\right)$ cross section for scattering off-shell gluons (off the virtual photon in the case of deep inelastic scattering).

Thus, for the structure function, $F_{L}\left(x, Q^{2}\right)$

$$
F_{L, N}\left(Q^{2}\right)=\left\langle e_{q}^{2}\right\rangle h_{L, N}\left(\gamma_{N}^{g g}\right) R_{N} f_{N}^{g}\left(Q^{2}\right)
$$

with

$$
h_{L, N}(\gamma)=\frac{\alpha_{s}}{2 \pi} N_{f} T_{R} \frac{4(1-\gamma)}{3-2 \gamma} \frac{\Gamma^{3}(1-\gamma) \Gamma^{3}(1+\gamma)}{\Gamma(2-2 \gamma) \Gamma(2+2 \gamma)}
$$

and where $\left\langle e_{q}^{2}\right\rangle$ is the mean quark charge squared. So, in order to evaluate $F_{L}\left(x, Q^{2}\right)$, we merely replace the $c_{n}$ coefficients in eq.(16) by the corresponding coefficients in the expansion of $h_{L, N}\left(\gamma_{N}^{g g}\right) R_{N}$ 
Similarly,

$$
h_{2, N}(\gamma)=\frac{2+3 \gamma-3 \gamma^{2}}{2(1-\gamma)} h_{L, N}
$$

determines the $Q^{2}$-dependence of $F_{2}\left(x, Q^{2}\right)$, i.e.

$$
\begin{aligned}
\frac{\partial F_{2, N}\left(Q^{2}\right)}{\partial \ln Q^{2}} & =\left\langle e_{q}^{2}\right\rangle\left[C_{g, N}^{(1)}\left(\alpha_{s}\left(Q^{2}\right)\right) \gamma_{N}^{g g}+2 n_{f} \gamma_{N}^{q g}\right] f_{N}^{g}\left(q^{2}\right) \\
& =\left\langle e_{q}^{2}\right\rangle h_{2, N}\left(\gamma_{N}^{g g}\right) R_{N} f_{N}^{g}\left(Q^{2}\right)
\end{aligned}
$$

to formally leading order.

In fig.(3), using the same choice of parameters (no more are needed) as in the discussion of $G\left(x, Q^{2}\right)$ previously, we present our predictions for the longitudinal structure function, $F_{L}\left(x, Q^{2}\right)$. As well as the full solution, we show the double log contribution to it. The largeness of the corrections to the double log calculation (in comparison to case of $G\left(x, Q^{2}\right)$ ), can be traced back to the fact that the second and third coefficients in the expansion of $h_{L, N} R_{N}$ are no longer zero. Also shown in fig.(3) is the result of re-fitting the HERA data on $F_{2}\left(x, Q^{2}\right)$, while keeping only the double log Bessel function. In order unambiguously to establish the existence of the high energy corrections, it is ultimately necessary to expose deviations from the double log approach (or more precisely approaches which do not sum the infinity of high order corrections $\left.\mathcal{O}\left(\alpha_{s} / N\right)\right)$ and so this is the reason for our comparison. As seen, the prediction from the double log approach is mostly larger than that for the full expression, but flatter with $x$. This largeness comes about mainly because the starting scale is much lower, and hence there has been more time for evolution to take place.

Let us now turn to the structure function, $F_{2}\left(x, Q^{2}\right)$ and its comparison with the HERA data [12]. We start by considering the expression of eq.(22). In order to construct $F_{2}\left(x, Q^{2}\right)$, we must integrate over $Q^{2}$ and invoke an input distribution, $F_{2}\left(x, Q_{0}^{2}\right)$. We choose this to be of the form $A+B x^{-\lambda}$. We see no reason to believe that the input form of $F_{2}\left(x, Q^{2}\right)$ should be purely flat since, as demonstrated, the gluon structure function always has some powerlike behaviour due to the coefficient function. Indeed, we are not able to obtain a very good fit with a completely flat input. We could have chosen an input of $B x^{-\lambda}$, and still obtain a comparable fit. However, our aim is not simply to obtain the best fit with the least number of parameters, but to determine the behaviour of the structure function as accurately as possible, and we believe the chosen input is the best way to do this. This introduces three extra parameters. The values of our 5 parameters $\left(Q_{0}\right.$, the normalisation of the input gluon density and the three parameters in $\left.F_{2}\left(x, Q_{0}^{2}\right)\right)$ are then obtained by fitting to the HERA data. Throughout, we 
work with 4 quark flavours and $\Lambda_{Q C D}=115 \mathrm{MeV}$. Agreement with the data is very good, i.e. $\chi^{2}=48$ for the 92 data points which have $x<0.01$.

Although we obtain very good agreement with the available HERA data, we expect our results to be subject to important corrections. Let us now explain why. In the leading log approximation, the structure function $F_{2}\left(x, Q^{2}\right)$ can be written in the form

$$
\begin{aligned}
F_{2, N}\left(Q^{2}\right) & =\left\langle e_{q}^{2}\right\rangle C_{g, N}^{(1)}\left(\alpha_{s}\left(Q^{2}\right)\right) f_{N}^{g}\left(Q^{2}\right)+\left[C_{q, N}^{(0)}+C_{q, N}^{(1)}\left(\alpha_{s}\left(Q^{2}\right)\right)\right] 2 n_{f}\left\langle e_{q}^{2}\right\rangle f_{N}^{s}\left(Q_{0}^{2}\right) \\
& +2 n_{f}\left\langle e_{q}^{2}\right\rangle C_{q, N}^{(0)} \int_{Q_{0}^{2}}^{Q^{2}} \frac{d q^{2}}{q^{2}}\left(\gamma_{N}^{q g} f_{N}^{g}\left(q^{2}\right)+\gamma_{N}^{q q} f_{N}^{s}\left(Q_{0}^{2}\right)\right) .
\end{aligned}
$$

The superscript on the coefficient functions specifies the order (in $\alpha_{s}$ ) of the contribution, i.e. $C_{i, N}=\sum_{n} C_{i, N}^{(n)}$. The parton model coefficient functions are $C_{g, N}^{(0)}=0$ and $C_{q, N}^{(0)}=1$.

Taking the derivative of this expression leads to eq.(22), but only after neglecting the higher order terms which are induced by differentiating the coefficient functions. Such terms are formally sub-leading since

$$
\frac{\partial}{\partial \ln Q^{2}}=-\alpha_{s}^{2} \frac{\beta_{0}}{4 \pi} \frac{\partial}{\partial \alpha_{s}}
$$

However, they are not sub-leading once eq.(22) is integrated to form the structure function, $F_{2}\left(x, Q^{2}\right)$.

To see how important these corrections are expected to be, we expand the coefficient function

$$
C_{g, N}^{(1)}=\sum_{n=0}^{\infty} p_{n} \bar{\alpha}_{s}\left(\frac{\bar{\alpha}_{s}}{N}\right)^{n} .
$$

The ratio of the term $\sim \bar{\alpha}_{s}\left(\bar{\alpha}_{s} / N\right)^{m}$ in $\partial C_{g, N}^{(1)} / \ln Q^{2}$ to the corresponding term in the series expansion of $C_{g, N}^{(1)} \gamma_{N}^{g g}$ is thus

$$
\left(-\frac{\beta_{0} \alpha_{s}}{4 \pi}\right) \frac{(m+1) b_{m}}{\sum_{i=0}^{m-1} b_{i+1} a_{m-i}} .
$$

Since $a_{n+1} / a_{n}=4 \ln 2$ for large $n$ and, assuming a similar relation for the $p_{n}$ coefficients, it follows that this ratio becomes

$$
\sim-\frac{\beta_{0} \alpha_{s}}{4 \pi} \frac{1}{A}
$$

where $a_{n} \approx A(4 \ln 2)^{n-1}$. Since $A \ll 1$ we cannot ignore such contributions. We should emphasise that $p_{n+1} / p_{n}$ cannot exceed $4 \ln 2$ (since we know the dominant singularity arises at $\left.N=4 \bar{\alpha}_{s} \ln 2\right)$ and that assuming any $p_{n+1} / p_{n}<4 \ln 2$ leads to an even stronger enhancement of the derivative terms (e.g. by a factor of $m$ for $p_{n+1} / p_{n} \ll 4 \ln 2$ ). All the evidence from the calculation of the series expansion of the coefficient function is that $p_{n+1} / p_{n}$ is indeed $\sim 4 \ln 2$ for large $n$. 
Although these corrections are formally sub-leading, we believe it is unlikely that they will cancel with higher order graphs and as such it is safe (and probably more appropriate) to include them in this order of the calculation. Since they lead to a negative contribution to $\partial F_{2}\left(x, Q^{2}\right) / \partial \ln Q^{2}$, we therefore expect a reduction in $\partial F_{2}\left(x, Q^{2}\right) / \partial \ln Q^{2}$ as $x$ falls at fixed $Q^{2}$ (or $Q^{2}$ falls at fixed and small $x$ ).

Using the expressions of ref.[1] we have computed the series expansions of the gluon coefficient function, $C_{g, N}^{(1)}$, and the anomalous dimension, $\gamma_{N}^{q g}$, in the $\overline{\mathrm{MS}}$ scheme to 18 th order (we will shortly have more to say on the choice of scheme), the first six terms of course agreeing with the numerical values explicitly given in ref. [1]. It is then a simple matter to calculate $F_{2}$ by transforming eq.(23) (using eq.(6) to give $f_{N}^{g}\left(Q^{2}\right)$ ) to $x$-space, in the same way that we obtained eq.(16) from eq.(12). We make the simplification that (as in eq.(16), and as when obtaining $F_{L}\left(x, Q^{2}\right)$ by transforming eq.(19) to $x$-space) we drop the quark singlet density in the definition of the gluon distribution (as explained in the remarks following eq.(6)). We also neglect $\gamma_{N}^{q q}$ and the $\mathcal{O}\left(\alpha_{s}\right)$ contributions to the quark coefficient function when calculating $F_{2}\left(x, Q^{2}\right)$ (since the input quark density is small in comparison to the evolved gluon density, the quark coefficient function is smaller than that for the gluon and $\gamma_{N}^{q q}$ is smaller than $\left.\gamma_{N}^{q g}\right)$. Using this method, we compute $F_{2}\left(x, Q^{2}\right)$ including those corrections which were neglected when eq.(22) was integrated over $Q^{2}$. The solid line in fig.(4) shows the result of a new fit to the HERA data and marginal improvement in the $\chi^{2}$ of the fit is found, i.e. $\chi^{2}=45$ for the 92 data points. A considerable improvement in the insensitivity to the value of $Q_{0}^{2}$ is also found. For our best fit $Q_{0}^{2}=2.0$ and $\mathcal{N}=1.1$, and these are the parameters used to determine our predictions for $G\left(x, Q^{2}\right)$ and $F_{L}\left(x, Q^{2}\right)$. We also find the input to $F_{2}\left(x, Q^{2}\right)$ to be $0.15+0.035 x^{-0.4}$.

From our results we conclude that our choice of a $\Theta$-function form of the gluon input is appropriate. Also, we note that the $\chi^{2}$ only starts to worsen significantly once $Q_{0}^{2} \lesssim 1 \mathrm{GeV}^{2}$. This is consistent with idea that the scale at which we define our input should be essentially arbitrary, providing it is large enough for the perturbative expansion to apply, and not too large to fill the available phase space. The dotted line in the figure shows the previously discussed best fit for $F_{2}\left(x, Q^{2}\right)$, i.e. ignoring the derivatives of the coefficient function. The dashed line shows the result of fitting the data taking only the leading term in the Bessel function expansion, i.e. the double log result, and flat inputs for the gluon and for $F_{2}\left(x, Q^{2}\right)$. This also has a very good $\chi^{2}$ of 44 for the 92 points, but high sensitivity to $Q_{0}^{2}$. It is clear from the plots that the differences between the full leading $\log$ calculation of $F_{2}\left(x, Q^{2}\right)$ and the dotted (and 
dashed) line are consistent with our expectations.

As a slight word of caution we must note that for low enough $x, \partial F_{2}\left(x, Q^{2}\right) / \partial \ln Q^{2}$ eventually becomes negative, and $F_{2}\left(x, Q^{2}\right)$ rises for falling $Q^{2}$. It is this region of negative $\ln Q^{2}$ derivative where our calculation starts to become untrustworthy. However, the effect only sets in for $x \sim 10^{-4}$ at $Q^{2}=Q_{0}^{2}$, and the value of $x$ at which $\partial F_{2}\left(x, Q^{2}\right) / \partial \ln Q^{2}=0$ falls very rapidly as $Q^{2}$ increases. Similarly, we feel that the predictions for the gluon and $F_{L}\left(x, Q^{2}\right)$ should be viewed with a little caution at extremely low $x$ and small $Q^{2}$. Nevertheless, it is reassuring that the region of breakdown is where we might expect physics beyond that considered in our approach to become important.

It is also important to comment on our choice of scheme. We could just as well have computed $F_{2}\left(x, Q^{2}\right)$ in the DIS scheme, and obtained precisely the same results as in $\overline{\mathrm{MS}}$ (see below). This is true providing we take care to include the sub-leading corrections to $\gamma_{N}^{q g}$ which contribute in the leading order to $F_{2}\left(x, Q^{2}\right)$. These terms are those neglected in eq.(5.27) of Catani and Hautmann when transforming to DIS scheme.

Before concluding, we wish to make a few remarks regarding the recent conclusions of Ellis et al 四]. Recall that $F_{2, N}\left(Q^{2}\right)-F_{2, N}\left(Q_{0}^{2}\right) \sim \alpha_{s} \mathcal{O}\left(\alpha_{s} / N\right)$, and as such any calculation which sums the leading logarithms must necessarily include the quark anomalous dimensions, $\gamma_{N}^{q g}$ (and $\gamma_{N}^{q q}$ ), computed to the same accuracy (i.e. $\sim \alpha_{s} \mathcal{O}\left(\alpha_{s} / N\right)$ ). Thus, it is not sensible to compute $F_{2, N}\left(Q^{2}\right)$ by including only the BFKL anomalous dimension, $\gamma_{N}^{g g} \sim \mathcal{O}\left(\alpha_{s} / N\right)$, and (for example) the two-loop form for the rest of the anomalous dimension matrix. It is also important to appreciate that, in the DIS scheme (where all the gluon coefficient functions are zero) the quark anomalous dimension $\gamma_{N}^{q g}$ is where the physics which gave the dominant (leading $\log )$ contribution to the gluon structure function $G\left(x, Q^{2}\right)$ resides, i.e.

$$
2 n_{f} \gamma_{N}^{q g}=R_{N} h_{2, N}\left(\gamma_{N}^{g g}\right)
$$

determines the DIS scheme anomalous dimension in terms of $R_{N}$ (calculated in $\overline{\mathrm{MS}}$ scheme). This is in contrast to the $\overline{\mathrm{MS}}$ scheme, where it is the coefficient function $C_{g, N}^{(1)}$ which has the large coefficients in the series expansion in $\bar{\alpha}_{s} / N$ (due to the corresponding large coefficients in $R_{N}$ ) and, as a result, the coefficients of $\gamma_{N}^{q g}$ are much smaller in the $\overline{\mathrm{MS}}$ scheme than in the DIS scheme. Therefore, the relatively small effect observed in ref. [U] when only the BFKL anomalous dimension is added to the full two-loop formalism is consistent with our finding that the $b_{i}$ coefficients in eq.(16) have little impact. However, on adding the all orders quark anomalous 
dimension, $\gamma_{N}^{q g}$, Ellis et al found huge effects which are very sensitive to how momentum conservation is applied. The largeness of their effect is driven by the largeness of the coefficients in the expansion of $\gamma_{N}^{q g}$ as calculated in the DIS scheme. We wish to urge caution in interpreting their results as evidence of important higher order corrections to the leading log BFKL framework. In particular, as we have just emphasised the bulk of the BFKL physics resides in the leading $\log$ factor $R_{N}$ (i.e. in the DIS scheme $\gamma_{N}^{q g}$ ), so part of the large effect which is seen after turning on $\gamma_{N}^{q g}$ is actually a leading effect. In addition, on adding the quark anomalous dimension the leading order expression or $F_{2}\left(x, Q^{2}\right)$ is given by eq.(23), whilst the next-to-leading order result is more complicated and involves, in particular, terms proportional to $\left(\gamma_{N}^{q g}\right)^{2}$. Such terms are small in the $\overline{\mathrm{MS}}$ scheme but may be large in DIS. The corresponding large terms never appear in a $\overline{\mathrm{MS}}$ scheme calculation since the coefficient function, $C_{g, N}^{(1)}$, is never iterated (i.e. raised to a power). Consistency between the schemes therefore suggests a cancellation of large terms in the DIS scheme. In order to perform a complete next to leading order calculation of $F_{2}\left(x, Q^{2}\right)$ there are many other terms which need to be computed, i.e. the $\sim \alpha_{s} \mathcal{O}\left(\alpha_{s} / N\right)$ corrections to the gluon anomalous dimensions $\gamma_{N}^{g g}$ (and $\gamma_{N}^{g q}$ ) and the higher order corrections to the quark anomalous dimensions, i.e. $\sim \alpha_{s}^{2} \mathcal{O}\left(\alpha_{s} / N\right)$. Cancellations should then occur between the terms proportional to the square of the leading order quark anomalous dimension and these other terms. By not performing the full next-to-leading calculation, Ellis et al are unable to observe these expected cancellations and as such their conclusions, based upon different assumptions regarding the imposition of momentum conservation, may be drawn prematurely.

\section{Conclusions}

We have presented an analytic approach to the evaluation of small $x$ cross sections and studied the behaviour of the gluon structure function, defined in a way which is consistent with the previous studies based upon the direct solution to the BFKL equation. In particular the solution in $x$-space is obtained exactly, and explicitly reveals the extreme limitations of the double leading log result and the power behaviour expected from the BFKL approach. In addition, we examined the deep inelastic structure functions $F_{2}\left(x, Q^{2}\right)$ and $F_{L}\left(x, Q^{2}\right)$ and demonstrated that the high energy corrections (to the double log calculation) are significant in the HERA region. Consistency with the data on $F_{2}\left(x, Q^{2}\right)$ is found. The structure function $F_{L}\left(x, Q^{2}\right)$ and the $Q^{2}$ dependence of $F_{2}\left(x, Q^{2}\right)$ should be able to provide sensitive tests of the small $x$ dynamics; in 
particular deviations from the traditional approach (expansion in $\alpha_{s}$ ) may well be observable.

We have not discussed the process of heavy quark production (in deep inelastic scattering or in photoproduction), although this process also ought to shed important light on the essential dynamics [8]. Also, the recent measurement by the ZEUS collaboration of the dijet cross section in photoproduction 13 could be confronted with theory using the techniques presented here [14. Finally, we wish to make available the expansion (in $\alpha_{s} / N$ ) of the coefficient function, $R_{N}$, and the $\overline{\mathrm{MS}}$ scheme expansions of the quark anomalous dimension $\gamma_{N}^{q g}$ and the gluon coefficient function $C_{g, N}^{(1)}$. These are displayed in the following table.

\section{Acknowledgments}

We wish to thank Keith Ellis, Francesco Hautmann and Graham Ross for helpful discussion. 


\section{Table}

Values of the first 18 coefficients in series expansions:

$$
\begin{gathered}
\gamma_{N}^{q g(\overline{\mathrm{MS}})}=\frac{\alpha_{s}}{2 \pi} \frac{1}{3} \sum_{n=0}^{\infty} a_{n}^{q g}\left(\frac{\bar{\alpha}_{s}}{N}\right)^{n}, \\
C_{g, N}^{(1)(\overline{\mathrm{MS}})}=\frac{\alpha_{s}}{2 \pi} n_{f} \frac{1}{3} \sum_{n=0}^{\infty} \tilde{p}_{n}\left(\frac{\bar{\alpha}_{s}}{N}\right)^{n}, \\
R_{N}^{(\overline{\mathrm{MS}})}=\sum_{n=0}^{\infty} \tilde{c}_{n}\left(\frac{\bar{\alpha}_{s}}{N}\right)^{n},
\end{gathered}
$$

i.e. $\tilde{p}_{n}=\frac{n_{f}}{18} p_{n}$ (see eq.(24)) and $\tilde{c}_{n} \bar{\alpha}_{s}^{n}=c_{n}$ (see eq.(15)).

\begin{tabular}{|r|c|c|c|}
\hline $\mathrm{n}$ & $a_{n}^{q g}$ & $\tilde{p}_{n}$ & $\tilde{c}_{n}$ \\
\hline 0 & 1.00 & 1.00 & 1.00 \\
1 & 1.67 & 1.49 & 0.00 \\
2 & 1.56 & 9.71 & 0.00 \\
3 & 3.42 & $1.64 \times 10^{1}$ & 3.21 \\
4 & 5.51 & $3.91 \times 10^{1}$ & -0.811 \\
5 & 7.88 & $1.29 \times 10^{2}$ & 4.56 \\
6 & $2.57 \times 10^{1}$ & $2.41 \times 10^{2}$ & $3.27 \times 10^{1}$ \\
7 & $4.42 \times 10^{1}$ & $6.53 \times 10^{2}$ & -2.95 \\
8 & $8.77 \times 10^{1}$ & $1.93 \times 10^{3}$ & $1.08 \times 10^{2}$ \\
9 & $2.83 \times 10^{2}$ & $4.01 \times 10^{3}$ & $4.00 \times 10^{2}$ \\
10 & $5.11 \times 10^{2}$ & $1.14 \times 10^{4}$ & $1.33 \times 10^{2}$ \\
11 & $1.24 \times 10^{3}$ & $3.17 \times 10^{4}$ & $2.10 \times 10^{3}$ \\
12 & $3.68 \times 10^{3}$ & $7.18 \times 10^{4}$ & $5.51 \times 10^{3}$ \\
13 & $7.17 \times 10^{3}$ & $2.07 \times 10^{5}$ & $5.30 \times 10^{3}$ \\
14 & $1.91 \times 10^{4}$ & $5.52 \times 10^{5}$ & $3.85 \times 10^{4}$ \\
15 & $5.29 \times 10^{4}$ & $1.33 \times 10^{6}$ & $8.49 \times 10^{4}$ \\
16 & $1.12 \times 10^{5}$ & $3.82 \times 10^{6}$ & $1.40 \times 10^{5}$ \\
17 & $3.11 \times 10^{5}$ & $1.00 \times 10^{7}$ & $6.95 \times 10^{5}$ \\
\hline
\end{tabular}




\section{References}

[1] S. Catani and F. Hautmann, Phys. Lett. B315 (1993) 157; Nucl. Phys. B427 (1994) 475.

[2] L.N. Lipatov, Sov. J. Nucl. Phys. 23 (1976) 338;

V.S. Fadin, E.A. Kuraev and L.N. Lipatov, Phys. Lett. B60 (1975) 50; Sov. Phys. JETP 44 (1976) 443; Sov. Phys. JETP 45 (1977) 199;

Y.Y. Balitsky and L.N. Lipatov, Sov. J. Nucl. Phys. 28 (1978) 822.

[3] R.D. Ball and S. Forte, CERN Preprint - Th/95-1, January 1995.

[4] R.K. Ellis, F. Hautmann and B.R. Webber, Phys.Lett. B348 (1995) 582.

[5] V.N. Gribov and L.N. Lipatov, Sov. J. Nucl. Phys. 15 (1972) 438;

L.N. Lipatov, Sov. J. Nucl. Phys. 20 (1974) 94;

G. Altarelli and G. Parisi, Nucl. Phys. B126 (1977) 298.

[6] T. Jaroszewicz, Phys. Lett. B116 (1982) 291.

[7] S. Catani, F.Fiorani, G. Marchesini and G. Oriani, Nucl. Phys. B361 (1991) 645; S. Catani, M. Ciafaloni and F. Hautmann, Phys. Lett. B242 (1990) 97; Phys. Lett. B307 (1993) 147; M. Ciafaloni, Nucl. Phys. B296 (1988) 49.

[8] S. Catani, M. Ciafaloni and F. Hautmann, Nucl. Phys. B366 (1991) 135.

[9] P.D.B. Collins and F. Gault, Phys. Lett. B112 (1982) 255; A. Donnachie and P.V. Landshoff, Nucl. Phys. B244 (1984) 322; Nucl.Phys. B267 (1986) 690.

[10] M. Glück, E. Reya and A. Vogt, Phys. Lett. B306 (1993) 391.

[11] R.D.Ball and S.Forte, Phys. Lett. B335 (1994) 77; Phys. Lett. B336 (1994) 77.

[12] ZEUS collaboration: M. Derrick et al, Zeit.Phys. C65 (1995) 379;

H1 collaboration: T. Ahmed et al, Nucl.Phys. B439 (1995) 471.

[13] ZEUS collaboration: M. Derrick et al, Phys.Lett. B348 (1995) 665.

[14] J.R. Forshaw and R.G. Roberts, Phys. Lett. B319 (1993) 539; Phys. Lett. B335 (1994) 494. 


\section{Figure Captions}

[1] Contour plot exhibiting the contribution to the full gluon structure function made by the double leading log term.

[2] Gluon structure function $G\left(x, Q^{2}\right)$ as a function of $x$ plotted for a range of $Q^{2}$ values. Contribution made by double leading log approximation shown by dot-dashed line.

[3] Prediction of longitudinal structure function $F_{L}\left(x, Q^{2}\right)$ as a function of $x$ plotted for a variety of $Q^{2}$ values. Contribution made by double leading log term shown by dot-dashed line. Also shown by dashed line is the prediction made using the best fit for $F_{2}\left(x, Q^{2}\right)$ while keeping only the double log term.

[4] Comparison of theoretical predictions with the small $x$ (i.e. $x<0.005$ ) data from the (a) ZEUS collaboration (renormalised up 2\%) and the (b) H1 collaboration (renormalised down 4\%). The dotted line corresponds to the best fit for this expression minus the formally subleading terms coming from the derivative of the coefficient function and the dashed line to the best fit for the double leading log approximation. 